\title{
Is that a bnik I see? Testing phonotactics using word- picture matching
}

\author{
Vsevolod Kapatsinski, Lamia Haddad Johnston \\ Department of Linguistics, University of Oregon, USA \\ https://doi.org/10.36505/ExLing-2010/03/0020/000140
}

\begin{abstract}
Phonotactic knowledge is typically tested using metalinguistic wordlikeness judgment tasks. We introduce a new method for testing phonotactics, where subjects are asked to match a set of pictures of novel objects with a larger set of pseudowords, only some of which are phonotactically legal. The subjects tend to pick the pseudowords that are phonotactically legal in their language and leave the illegal ones unassigned. We compare the results of the word-picture matching to a traditional rating task and show that the two tasks produce somewhat different results despite sharing some $(25 \%)$ of the variance. We argue for methodological pluralism in the study of phonotactics.
\end{abstract}

Key words: phonotactics, word-picture matching, rating.

\section{Introduction}

Speakers' knowledge of the phonotactic constraints of their language affects the development of the lexicon in the domains of borrowing, coining, and word retention over time. This can happen through listeners misperceiving phonotactically illegal words as similar-sounding legal words (Berent et al. 2007, Ohala 1981) or speakers mispronouncing phonotactically illegal words. In addition, even if a word can be correctly perceived and produced, speakers may minimize effort by choosing to produce or adopt phonotactically legal words over phonotactically illegal competitors (Berg 1998, Martin 2007, Schwartz \& Leonard 1982).

The main method for testing phonotactic knowledge, at least in adults, is a rating task, where subjects are asked to judge the acceptability or 'wordlikeness' of various pseudowords (e.g., Bailey \& Hahn 2001, Frisch et al. 2000, Shademan 2005, Treiman et al. 2000). Although recent studies have developed methods for examining the effect of phonotactics on misperception (Berent et al. 2007), the effect of phonotactics on lexical choice remains unexplored experimentally. In this paper, we propose one method (word-picture matching) for testing an influence of phonotactics on lexical choice. We ask subjects to select from a set of words and assign them to a smaller set of referents. While requiring much more time than a ratings task, the method avoids explicit solicitation of phonotactic judgments and provides a way to examine other potential factors that influence lexical choice, including sound symbolism (Sapir 1929) and lexical

ExLing 2010: Proceedings of 3rd Tutorial and Research Workshop on Experimental Linguistics, 25-27 August, Athens, Greece 
analogy (Bailey \& Hahn 2001, Shademan 2005). Using the same set of stimuli, we will compare the results of a ratings task with our novel pictureword matching paradigm.

\section{Methods}

40 native English speakers were presented with a series of Powerpoint slides. The first slide contained a short background story and instructions. The story asked subjects to imagine arriving on another planet in the distant future and discovering the remains of an Earth colony established by speakers of English and Wilkipaengo (an invented language name). They were told that they would hear lists of both English and Wilkipaengo names for creatures along with creatures' pictures and they were instructed to assign English words to the creatures.

The following six slides each contained 6 creatures and 12 sound files of pseudowords (6 legal and 6 illegal, equally balanced between /i/ and $/ \mathrm{a} /$ arranged in a column on the left side of the slide) that the subjects could listen to as many times as necessary by double-clicking on them. The subjects assigned a name to a creature by dragging a sound file icon on top of the chosen creature. Thus, we were able to avoid presenting spellings of the words.

For each phonotactically illegal word, there was a corresponding phonotactically legal word. The two words in a pair differed from each other only by the onset, which did or did not contain an illegal cluster (e.g., blick/bnick, fnek/frek, sren/fren). The two words in a pair were presented to different subjects but on the same slide and in the same location within the slide.

An additional group of 23 native English speakers were asked to rate the same stimuli on a scale from 1-5 for how much each word sounded like a typical English word. If the word sounded completely normal, the subjects were to pick 5 , if it sounded "totally weird", they were to pick 1 .

\section{Results}

Phonotactically legal words were significantly more likely to be assigned to creatures than the corresponding phonotactically illegal words: $\mathrm{t}(71)=8.05, \mathrm{p}<.0001$ (in Figure 1, there are more words above the horizontal axis than below it), and were rated as sounding more like typical English words: $\mathrm{t}(71)=5.09, \mathrm{p}<.0001$ (in Figure 1, there are more words to the right of the vertical axis than to the left of it).

Figure 1 shows that there is a highly significant correlation between popularity in being assigned to creatures and being rated as sounding typically English $(\mathrm{r}=.5, \mathrm{t}(70)=4.78, \mathrm{p}<.0001)$. However, the correlation explains only $25 \%$ of the variance, leaving $75 \%$ of the variance between tasks unaccounted for. We tested whether such a difference would be observed 
different groups of subjects exposed to the same task using resampling. We drew a random sample of 10 subjects from a population of 20 subjects with the same distribution of scores as in each of our actual samples of subjects exposed to a certain set of stimuli in a certain task. We then correlated the scores for words across subject samples within a task and across tasks. The correlation coefficients between samples that came from within the same task were reliably higher than the coefficients for samples coming from different tasks $(\mathrm{p}<.001)$, indicating that the two tasks reliably differ in which words they favor. Based on the confidence interval on the difference between within-task and across-task correlation coefficients, at least $4 \%$ of the variance in the data is due to the difference between tasks rather than random between-subject differences (compared to $25 \%$ of shared variance across tasks explainable by phonotactics).

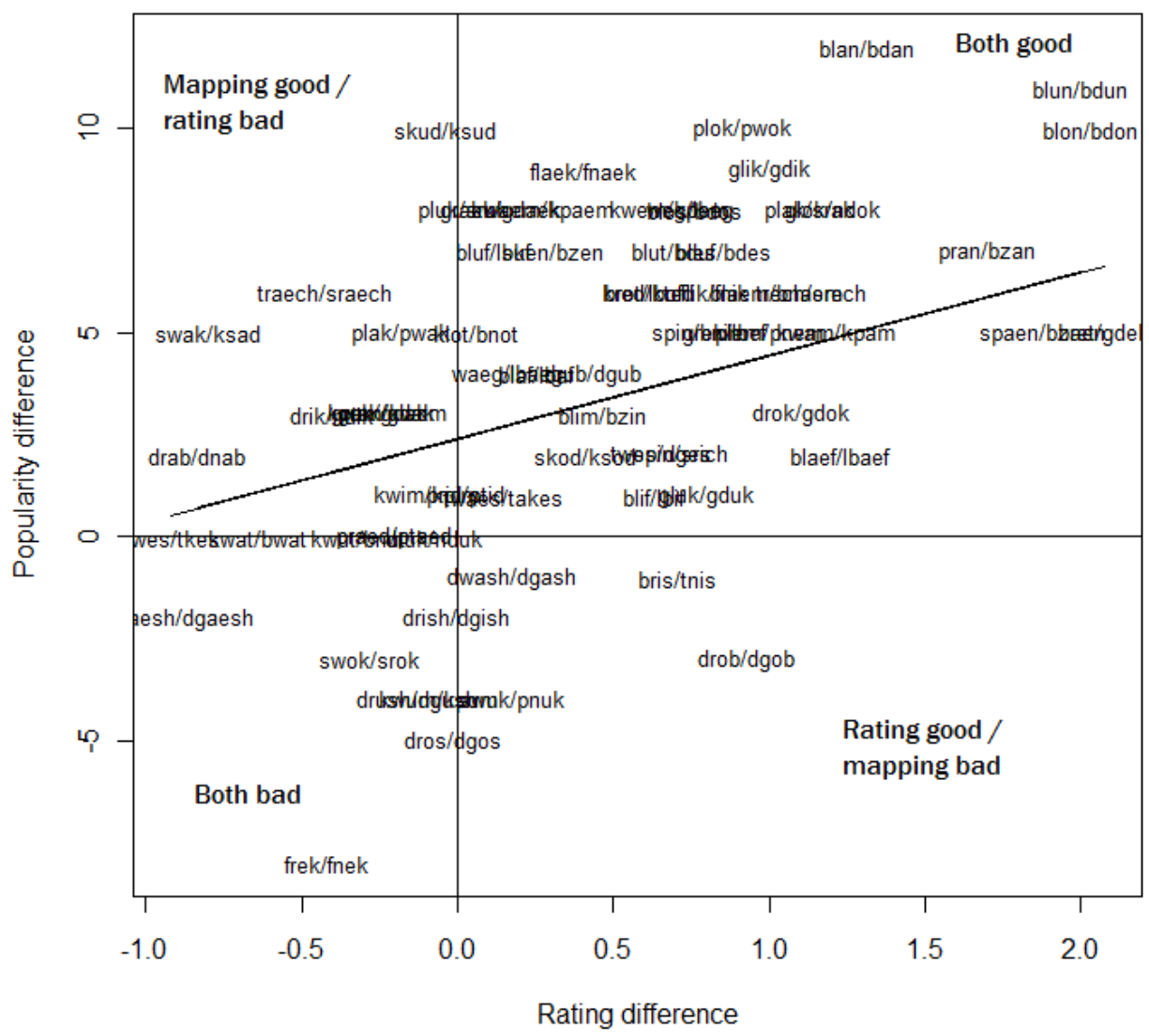

Figure 1. Standardized ratings vs. popularity in word-picture mapping.

Overall, the two tasks are comparable in the number of word pairs in which the illegal word is unexpectedly preferred to the legal one. In both tasks, such 
exceptional words tend to involve legal \#Cw clusters, which are very low in type frequency in English, and \#dr clusters, which are strongly affricated in the stimuli and may be perceived as the illegal \#dzr by the participants who do not affricate as much. In addition, the exceptional preference for /fnek/ (the most popular and highly-rated illegal word) over /frek/ appears to be due to lexical analogy. While this would just be speculation based on rating data, the mapping task allows us to test for non-random picture-word co-occurrences. In this experiment, /fnek/ was mapped onto the only horizontally elongated legless creature on the slide, thus its high popularity and ratings are likely due to the word 'snake'.

\section{Conclusion}

We confirm English speakers' sensitivity to the phonotactics / frequency of occurrence of onset clusters in a novel task, auditory word-picture matching, which allows experimental testing for the influence of the subjects' phonotactic knowledge on lexical choice and provides possible explanations for unexpected results due to lexical analogy. A word that is phonologically similar to an existing word in English, may be rated as acceptable, even if a portion of the word violates English phonotactics; analogies that are unclear in a ratings class can surface when we examine the assigned referents in a word-picture matching task.

\section{References}

Bailey, T., Hahn, U. 2001. Determinants of wordlikeness: Phonotactics or lexical neighborhoods? Journal of Memory \& Language 44, 568-591.

Berent, I., Steriade, D., Lennertz, T., Vaknin V. 2007. What we know about what we have never heard. Cognition 104, 591-630.

Berg, T. 1998. Linguistic Structure and Change. Oxford University Press.

Frisch, S. A., Large, N. R., Pisoni, D. B. 2000. Perception of wordlikeness. Journal of Memory \& Language 42, 481-496.

Martin, A. 2007. The Evolving Lexicon. PhD Dissertation, UCLA.

Ohala, J.J. 1981. The listener as a source of sound change. Chicago Linguistic Society 17-2, 178-203.

Sapir, E. 1929. A study in phonetic symbolism. J. of Exper. Psychology 12, 225-239.

Schwartz, R. G., Leonard, L. B. 1982. Do children pick and choose? Journal of Child Language 9, 319-336.

Shademan, S. 2005. Is phonotactic knowledge grammatical knowledge? Proceedings of the West Coast Conference on Formal Linguistics 25, 371-379.

Treiman, R., Kessler, B., Knewasser, S., Tincoff, R., Bowman, M. 2000. English speakers' sensitivity to phonotactic patterns. Laboratory Phonology 5, 269-282. 